\title{
Study on the effect of ambient gas on nanostructure formation on metal surfaces during femtosecond laser ablation for fabrication of low-reflective surfaces
}

\author{
Tomi SMAUSZ ${ }^{\mathrm{a}, \mathrm{b}, *}$, Tamás CSIZMADIA ${ }^{\mathrm{b}, \mathrm{c}}$ Csaba TÁPAI ${ }^{\mathrm{b}}$, Judit KOPNICZKY ${ }^{\mathrm{b}}$, Albert OSZKÓd, Martin \\ EHRHARDT $^{\mathrm{e}}$, Pierre LORENZ ${ }^{\mathrm{e}}$, Klaus ZIMMER ${ }^{\mathrm{e}}$, Andrea PRAGER ${ }^{\mathrm{e}}$, Béla HOPP \\ ${ }^{a}$ MTA-SZTE Research Group on Photoacoustic Spectroscopy, University of Szeged, 6720 Szeged, Dóm \\ tér 9, Hungary \\ ${ }^{b}$ Department of Optics and Quantum Electronics, University of Szeged, H-6720 Szeged, Dóm tér 9 , \\ Hungary \\ ${ }^{c}$ Attosecond Light Pulse Source, ELI-Hu Nkft, H-6720 Szeged, Dugonics ter 13, Hungary \\ ${ }^{d}$ Department of Physical Chemistry and Material Science, University of Szeged, H-6720 Szeged, Aradi \\ vértanuk tere 1, Hungary \\ ${ }^{e}$ Leibniz-Institut für Oberflächenmodifizierung e. V., Permoserstr. 15, 04318 Leipzig, Germany \\ "corresponding author: tomi@physx.u-szeged.hu
}

Keywords: black metal; low reflectivity; femtosecond laser; nanostructure; gas environment 


\begin{abstract}
Nanostructure formation on bulk metals (silver, gold, copper and titanium) by femtosecond Ti-sapphire laser irradiation $(775 \mathrm{~nm}, 150 \mathrm{fs})$ is studied aiming the production of low-reflectivity surfaces and the better understanding of the development process. The experiments were performed in nitrogen, air, oxygen and helium environments at atmospheric pressure. The samples were irradiated with fluences in the $0.1-2 \mathrm{~J} / \mathrm{cm}^{2}$ range and an average pulse number of 100 falling over a given area. The reflectivity of the treated surfaces was determined by a microspectrometer in the $450-800 \mathrm{~nm}$ range and their morphology was studied by scanning electron microscopy. The gas ambience influenced the results via two effects: formation processes and the chemically-induced modifications of the nanostructures. In case of He the nanoparticle aggregates - otherwise generally present - are predominantly missing, which leads to a lower darkening efficiency. The presence of oxygen enhances the darkening effect for copper mostly at lower fluences, while causes a slow increase in reflectivity in the case of titanium (in case of pure oxygen) in the high fluence range. The surface morphology in case of nitrogen and air were quite similar probably due to their close molecular mass values.
\end{abstract}




\section{Introduction}

Pulsed laser processing technologies are advanced tools in material processing and micromachining, which have already demonstrated their ability to produce random nano- and microstructures on the surface of numerous metals [1-3]. One important aspect of these laser irradiation induced surface textures is that they can exhibit modified optical properties [4-6]. Spectroscopic studies showed that originally highly reflective materials may become almost completely absorptive in a broad spectral range (from near UV to near IR) due to the nanopatterning of the treated sample surface. Possible applications of these broadband absorptive metal surfaces cover every field which needs efficient light trapping and manipulation techniques for example the development of antireflection coatings, stealth technology, metal colorization, optoelectronics, solar light absorbers, broadband thermal sources or improving power conversion efficiencies in photovoltaic devices generally [7-9].

Albeit several papers have been presented so far that aim the description of the optical property modifications in correlation with the changes of the surface morphology of metals in case of femtosecond and picosecond laser pulses [10-15], these experiments were mainly carried out in the presence of atmospheric air environment. However it is known that the darkening effect is not only attributed to the light trapping properties of the rough surface, but in certain cases oxidation and other chemical modification of the illuminated surfaces can also play an important role $[14,16]$. Guan et al. studied color change of different material surfaces following laser irradiation, and they found that it resulted from the controlled surface oxidation during laser beam interaction with metal surfaces $[17,18]$. In the experiments of Qian et al. different oxide layers were formed on $\mathrm{KrF}$ excimer laser-irradiated Ti surface, and they proposed that not only the morphological change, but the compositional variations of the irradiated area also play an important role in the laser-induced coloration [19].

In this paper, we study the impact of gas environment on the laser induced surface darkening process of different target materials. The comparison of the results published in the literature are often hardly comparable due to the different experimental arrangements and parameter sets, therefore our aim was to perform a comprehensive study using various target materials and gas ambiences while keeping the same experimental conditions during the irradiations. The applied ambient gases (nitrogen, air, oxygen and helium) and target materials (silver, gold, copper and titanium) represent a variety of physical and chemical properties. The motivation of the current research is to answer open questions regarding the mechanism of reflectivity decrease and surface structure formation throughout the darkening process, 
while exploring the role of the gas environment during femtosecond laser irradiation. Particular attention was paid to the morphological studies of the irradiated areas.

\section{Experimental}

In this study high purity non-polished metal (silver, gold, copper and titanium) plates (from Goodfellow) were irradiated directly by pulses of a Ti-sapphire laser (CPA-2001, Clark MXR: $\lambda=775 \mathrm{~nm}, \tau_{\mathrm{FWHM}}=150 \mathrm{fs}, f=1 \mathrm{kHz}$ ) in different gas environments (nitrogen, air, oxygen and helium) at atmospheric pressure. The laser beam was focused onto the sample surface in a circular spot with $152 \mu \mathrm{m}$ diameter and the targets were scanned over $1 \mathrm{~mm} \times$ $1 \mathrm{~mm}$ sized area by a 4-pole magnet motor driven XY translator. The scanning speed was kept constant and each scanned row was shifted to the previous one by $50 \%$ of the laser spot diameter ensuring an average 100 pulses on a given area. The laser fluence was varied between 100 and $2000 \mathrm{~mJ} / \mathrm{cm}^{2}$.

\section{Results}

\subsection{Reflectivity measurements}

After laser processing, the darkening of the treated surfaces was perceptible to the naked eye for all experimental condition applied. The specular reflectivity of the treated areas were measured in the visible-near-infrared spectral range $(450-800 \mathrm{~nm})$ by a microspectrometer equipped with an $N A=0.12$ objective. The detected light was reflected/backscattered from a circular area having a diameter of less, than $100 \mu \mathrm{m}$. The recorded spectra were normalized to the reflectivity of the untreated, non-polished metal samples measured with the same setup. Figure 1 shows the dependence on the laser fluence of the normalized reflectivities of silver (a), gold (b), copper (c), and titanium (d) samples at $\lambda=550 \mathrm{~nm}$ in various gas environments. It was found that the normalized reflectivity decreased rapidly with increasing fluence, reaching a final value below 0.05 in most cases. Exceptions were observed when performing irradiation in He environment or in the case of titanium when the ambience contained oxygen (air or pure oxygen).

\section{Silver}

The drop of the reflectivity was the fastest in case of silver, where a value below 0.05 was reached already at $500 \mathrm{~mJ} / \mathrm{cm}^{2}$ in all the applied gas environments. At higher fluences only a slight further reduction can be observed in the measured reflectivity. The tendencies were quite similar in air, $\mathrm{N}_{2}$ and $\mathrm{O}_{2}$ environments while the decrease was slower when using He. 


\section{Gold}

In case of gold the tendencies of the fluence dependence are quite similar to that of silver but with a less steep decrease of the relative reflectivity.

\section{Copper}

For copper targets the normalized reflectivities at $100 \mathrm{~mJ} / \mathrm{cm}^{2}$ have high uncertainty due to the surface cleaning effect observed at this low fluence value. At higher fluences the oxidation of the surface [20] enhances the darkening effect of the laser leading to a steeper decrease of the normalized reflectivity. The use of He gas resulted in significantly higher reflectivities in the whole studied fluence range as compared to the other gases.

\section{Titanium}

The behavior of the titanium was different from the other three metals. In air, $\mathrm{N}_{2}$ and $\mathrm{O}_{2}$ ambience the relative reflectivity decreased below 0.1 already at $200 \mathrm{~mJ} / \mathrm{cm}^{2}$. Increasing the fluence above $500 \mathrm{~mJ} / \mathrm{cm}^{2}$ in nitrogen atmosphere the reflectivity decreased further, however, in case of air the reflectivity remained constant. In pure oxygen the measurements clearly indicated an increasing reflectivity when increasing the fluence above $200 \mathrm{~mJ} / \mathrm{cm}^{2}$. When looking at the samples with unaided eye in an order of increasing fluences, the initial darkening turned into lighter and lighter gray shades. The tendencies of the data plots obtained for air environment fell between the corresponding ones obtained for nitrogen and oxygen, the two main constituents of air. In He environment the darkening efficiency of laser irradiation was reduced as compared to air.

\subsection{Morphological investigations}

To explain the results presented above, we investigated the morphology of the laserirradiated metal samples. The developed surface structures were observed by a Hitachi S4700 scanning electron microscope (SEM) operating at $20 \mathrm{kV}$ accelerating voltage and $10 \mu \mathrm{A}$ emission current without applying any additional conductive coating on the investigated surfaces. Since the observed characteristic structures were in each case very similar in air and nitrogen environments, the latter ones are not presented in the forthcoming figures.

\section{Silver and gold}

The surface structures on silver formed by irradiation at various fluences are shown in Figure 2. It was found that significant morphological changes occur even at $200 \mathrm{~mJ} / \mathrm{cm}^{2}$ in air, $\mathrm{N}_{2}$ and $\mathrm{O}_{2}$ atmosphere. At the highest applied fluence $\left(F=2 \mathrm{~J} / \mathrm{cm}^{2}\right)$ various surface structures developed: in air and nitrogen environment typically molten and refrozen jets and droplets can be seen partially covered by filament-like nano-aggregates (approximately few-nanometers in 
size), while in oxygen the laser-induced periodic surface structures (LIPSS) - ripples -appear with less than $1 \mu \mathrm{m}$ spatial period under the droplets and nano-aggregates. The diameters of these droplets vary in approximately $100 \mathrm{~nm}-1000 \mathrm{~nm}$ range. In SEM figures larger field of view inserts were used in case when existing LIPPS were not clearly visible on $50 \mathrm{kX}$ magnification. These low-spatial-frequency LIPPS structures (LSFL) with spatial periods slightly smaller than or comparable to the laser irradiation wavelength is induced by the optical interference of the incident laser radiation with the surface electromagnetic wave generated on the rough surface by the laser irradiation [21,22]. When irradiating the silver target in He environment the surface structures formed are very different from those obtained in other gases. LSFLs are visible at $200 \mathrm{~mJ} / \mathrm{cm}^{2}$ applied fluence and vanish at higher fluences. While the surface becomes irregular, the larger molten droplets and nano aggregates with filament structures, which were present in case of the other gases are completely missing in He. In case of gold the characteristic surface structures were similar to those observed for silver.

\section{Copper}

Figure 3 reveals that the features on the irradiated surface of copper are similar to the structures observed on silver irradiated in air and $\mathrm{N}_{2}$. In $\mathrm{O}_{2}$ and $\mathrm{He}$ the periodic surface structures are evident at all fluences in the investigated range. Nano-aggregates and droplets were observed in oxygen and air at high fluences only, but in much smaller extent than for silver and gold.

\section{Titanium}

Figure 4 shows the morphology of titanium irradiated in different gas environments. Using air and nitrogen gases the surface becomes densely covered by complex filament-like formations even at $200 \mathrm{~mJ} / \mathrm{cm}^{2}$ laser fluence and more or less maintain its morphology until the highest applied fluence $\left(F=2000 \mathrm{~mJ} / \mathrm{cm}^{2}\right)$. In oxygen a considerable amount of filament like structures are deposited at higher fluences, which cover up the laser induced periodic surface structures clearly existing at $200 \mathrm{~mJ} / \mathrm{cm}^{2}$. In contrast, much more conglutinated, molten and resolidified structures can be found at the low fluence regime (below $500 \mathrm{~mJ} / \mathrm{cm}^{2}$ ) in helium, which are gradually covered by nano-aggregates at high fluences. On the hill of the periodic surface structures of $\mathrm{Ti}$, small spheres (microdots) can be discovered, which were also observed by Tsukamoto et al. during stationary irradiation of $\mathrm{Ti}$ at similar laser parameters $[23]$.

\subsection{Chemical compsition analysis}


The chemical composition of the samples was studied by X-ray photoelectron spectroscopy (XPS, SPECKS and AXIS ULTRA) and energy dispersive X-ray spectroscopy (EDX, Röntec XFlash attached to the SEM).

Compared to the non-treated sample, chemical changes were observed for silver samples irradiated in oxygen containing ambience. The appearance of Ag3d peak at $367.7 \mathrm{eV}$ indicated the formation of $\mathrm{Ag}_{2} \mathrm{O}$ on the surface. In case of gold, the disappearance of $\mathrm{Au} 4 \mathrm{f}$ peak at $85.7 \mathrm{eV}$ indicated even the removal of the initially present small amount of oxide from the target surface independently of the gas environment. In case of copper, the formation of $\mathrm{CuO}$ was detected on the surface after the irradiation in air. The amount of $\mathrm{CuO}$ increased further when the irradiation was performed in pure oxygen. However, the irradiation of copper in pure nitrogen resulted in the complete disappearance of the $\mathrm{CuO}$ peak at $933.6 \mathrm{eV}$ binding energy, and instead, an increased nitrogen content was detected. In case of titanium, the $\mathrm{Ti}^{\mathbf{4}}\left(\mathrm{TiO}_{2}\right)$ peak at $458.8 \mathrm{eV}$ was observed on all samples regardless of the applied ambient gas, and it was seen even on the untreated areas. This could be explained by the presence (or prompt formation) of native $\mathrm{TiO}_{2}$ layer on the surface of titanium, and the shallow detection depth of the XPS method. Therefore, we applied an alternative analytical method, EDX analysis as well. The excitation depth $(\sim 1 \mu \mathrm{m})$ during EDX is comparable to the thickness of the processed volume of the. According to the EDX data the oxygen content of the irradiated surface volumes strongly depends on the chemical nature of the applied gas ambience. The average oxygen-to-titanium atomic ratio (O:Ti) obtained on the sample prepared in $\mathrm{He}$ at the highest fluence was somewhat lower $(\mathrm{O}: \mathrm{Ti}=0.2 \pm 0.04)$ than that obtained on the untreated reference area $(\mathrm{O}: \mathrm{Ti}=0.25 \pm 0.05)$, while a much higher oxygen content was detected on samples irradiated in air $(\mathrm{O}: \mathrm{Ti}=1.9 \pm 0.35)$ and pure oxygen $(\mathrm{O}: \mathrm{Ti}=2 \pm 0.4)$.

Table 1. summarizes the main observations for the different target-gas combinations in terms of surface morphology (formation of basic nanostructure, presence of LIPPS and deposition of nano-aggregates), and chemical changes as well as their possible effect on the measured reflective properties.

\begin{tabular}{llllll} 
& & $\mathbf{A g}$ & $\mathbf{A u}$ & $\mathbf{C u}$ & $\mathbf{T i}$ \\
\hline \multirow{2}{*}{ Air } & morphology & -basic nanostructure & -basic nanostr. & -basic nanostr. & -basic nanostr. \\
& & -nano-aggregates & -nano-aggregates & - nano-aggregates & -intensive nano-aggr. \\
\cline { 2 - 6 } & chemistry & -no changes & -no changes & -oxidation & $\begin{array}{l}\text {-oxidation decreases } \\
\text { darkening efficiency }\end{array}$ \\
\hline $\mathbf{N}_{\mathbf{2}}$ & morphology & -basic nanostr. & -basic nanostr. & -basic nanostr. & -basic nanostr. \\
& & -nano-aggregates. & -nano-aggregates. & -lower nano-aggr. & -intensive nano-aggr. \\
\cline { 2 - 6 } & chemistry & -no changes & -no changes & built-in nitrogen & -no changes \\
\hline $\mathbf{O}_{2}$ & morphology & -basic nanostr. & -basic nanostr. & -basic nanostr. & -basic nanostr. \\
& & -LIPPS & -LIPPS & -LIPPS & -LIPPS \\
\hline
\end{tabular}




\begin{tabular}{llllll}
\hline & & -nano-aggregates & -lower nano-aggr. & -low nano-aggr. & -intensive nano-aggr. \\
\cline { 2 - 6 } & chemistry & - oxidation & -no changes & $\begin{array}{l}\text {-oxidation fastens } \\
\text { the darkening }\end{array}$ & $\begin{array}{l}\text {-oxidation decreases } \\
\text { darkening efficiency }\end{array}$ \\
\hline He morphology & -less significant basic & -less significant & -less significant & -basic nanostr. \\
& & nanostr. & basic nanostr. & basic nanostr. & -LIPPS \\
& & -missing nano-aggr. & -LIPPS & -LIPPS & -nano-aggregates \\
& -less darkening & -missing nano-aggr. & - missing nano- & -less darkening \\
& efficiency at lower & -less darkening & aggr. & efficiency \\
& fluences & efficiency at lower & -less darkening & \\
& & fluences & efficiency at lower & \\
& & & fluences & \\
& & -no changes & -no changes & -no changes
\end{tabular}

Table 1: Summary of the most important observations for the different target-gas combinations.

\section{Discussion}

Laser ablation influenced mostly by the thermal and optical properties of the target material is the first stage in the formation of the observed nanostructures. The formation of an intensive plume accompanied by direct ejection of nanofragments from the surface occurs in this step. The resulting basic surface structures greatly affect the reflective properties of the irradiated area. The expansion of the plume and its interaction with the ambient gas is the second stage, when nanoparticles are condensed and partly deposited onto the surface also contributing to the terrain formation. The chemical interaction between the plume and the ambient gas can be regarded as a third factor influencing the reflective properties of the treated surfaces. Therefore, we discuss the obtained results according to the above partition.

\subsection{The ablation stage}

Although all the investigated metals are in the d-block of the periodic table, the electron configuration and therefore the physical properties of $\mathrm{Ti}$ is remarkably different from the other three elements $(\mathrm{Cu}, \mathrm{Ag}, \mathrm{Au})$ in group 11 (see Table 2). The laser ablation process and the nanostructure formation is strongly affected by the reflectivity, the electron-phonon coupling constant and the thermal conductivity of the target material [24]. Laser ablation begins with the absorption of the laser pulse energy by accelerating the conduction band electrons of the target metal. The absolute reflectivity of the intact plane Ti surface is much lower than the initial reflectivity of the other three investigated metals (Table 2), which resulted in higher absorbed energy during the first few pulses. The laser absorption induced excitation of the conducting electrons is followed by the energy transfers from these electrons to the vibrational modes of the crystal lattice. The efficiency of this process is characterized 
by the so-called electron-phonon coupling constant. By this constant, the volume of the heated material and also the temperature distribution throughout that volume can be defined. The thermal conductivity and the specific heat of the target metal determines the lifetime of the molten phase both in the irradiated surface area and in the nanoparticles being formed and thereby the size distribution and the aggregation extent of the formed nanodroplets. In case of $\mathrm{Ti}$, the lowest reflectivity and thermal conductivity in conjunction with the highest electronphoton coupling constant and specific heat resulted in much stronger nanoparticle aggregation and redeposition of the filament-like structures onto the titanium surface as compared to the other three examined metals as it is indicated in figure 4.

\begin{tabular}{|c|c|c|c|c|}
\hline & $\mathrm{Ti}$ & $\mathrm{Cu}$ & $\mathrm{Ag}$ & $\mathrm{Au}$ \\
\hline $\begin{array}{l}\text { Original reflectivity (\%) } \\
\text { (at } 775 \mathrm{~nm} \text { ) }\end{array}$ & 62.2 & 96.2 & 97.2 & 96.8 \\
\hline $\begin{array}{l}\text { Thermal conductivity } \\
(\mathrm{W} / \mathrm{mK})\end{array}$ & 22 & 401 & 429 & 310 \\
\hline Atomic mass & 47.87 & 63.55 & 107.87 & 196.97 \\
\hline Density $\left(\mathrm{g} / \mathrm{cm}^{3}\right)$ & 4.51 & 8.96 & 10.5 & 19.3 \\
\hline $\begin{array}{l}\text { Electron-phonon coupling } \\
\text { constant }[25]\end{array}$ & 0.38 & 0.13 & 0.12 & 0.17 \\
\hline Specific heat (J/kgK) & 544.3 & 376.8 & 238.6 & 125.6 \\
\hline $\begin{array}{l}\text { Standard electrode potential } \\
\text { (V) }\end{array}$ & $-1.63[26]$ & $0.337[27]$ & $0.799[28]$ & 1.83 [29] \\
\hline
\end{tabular}

Table 2: Important physical and chemical properties of the investigated metals.

\subsection{Plume expansion dynamics and nanoparticle formation}

The results show that the development of morphological structures and the change in the reflective properties of the target sample are influenced by the nature of the ambient gas in which the irradiation occurs. The nucleation becomes significant after the thermalisation of the one-component plume, while upon mixing with the ambient gas a diffusion driven nucleation, condensation (growth) and aggregation starts. Generally, the nanoparticle and aggregate formation needs high plasma/nanoparticle density and therefore it is strongly influenced by the plume expansion dynamics in the different gas environments [30,31]. S.B. Wen et al. [32] studied the nanoparticle generation during copper ablation in atmospheric argon and helium ambients and compared the experimental results with the numerical gas dynamics simulations of the nanoparticle condensation process. Although the study was made for nanosecond pulses, the main conclusions on the plume expansion dynamics and the 
interaction with the ambient gas can stand for our case, too. The particle condensation process and the particle transport showed significant differences for $\mathrm{Ar}$ and $\mathrm{He}$ atmospheres. In the low molecular mass $\mathrm{He}$, which possess high thermal conductivity and high diffusion coefficient, the condensation process starts and finishes earlier than in Ar ambient, and smaller nanoparticles are formed. Moreover, in helium, the nanoparticles are more efficiently mixed with the ambient atmosphere and transported away from the ablation site, while in argon, a significant amount of nanoparticles falls back onto the surface. Since the critical physical properties of the ambient gases are strongly related to their atomic or molecular masses (e.g., 4 and 39.94 for the atomic masses of He and Ar, and 28.97, 28 and 32 for the molecular masses of Air, $\mathrm{N}_{2}$ and $\mathrm{O}_{2}$ respectively), great differences can be expected in the nanoparticle formation processes in the different atmospheres. In accordance with this, much faster expansion of the plasma plume occurs in He inhibiting the nanoparticle creation, agglomeration and deposition onto the target surfaces. In He only the relatively low atomic mass titanium showed noticeable nano-aggregate deposition at the highest fluence applied.

\subsection{Effects of chemical reactions during irradiation}

The XPS and EDX measurements indicated that in case of silver chemical changes had a minor contribution to the darkening process while in the case of titanium and copper, the two metals with relatively high reducing ability (i.e. low standard electrode potentials, see Table 2), a much stronger chemical influence can be seen. In case of gold no chemical compound formation was detected independently of the gas environment. When irradiating silver, the oxygen content of the applied gas ambience promoted the formation of black colored $\mathrm{Ag}_{2} \mathrm{O}$ on the surface, however it did not seem to have a significant contribution to the darkening process. In case of copper irradiation in air the formation of $\mathrm{CuO}$ accelerates the darkening process as demonstrated in earlier studies [14,16], too. The oxidation effect could be further enhanced when irradiating the sample in pure oxygen atmosphere. When performing the irradiation in pure nitrogen the oxide formation was evidently missing, while an increased nitrogen content was detected. In case of titanium the presence of oxygen initiated the increase of the (diffuse) reflectivity of the target when increasing the fluence in the elevated fluence range. This observation could be explained by the formation of white color titanium dioxide.

\section{Summary}


The influence of the ambient gas on laser induced darkening of various metals (copper, silver, gold and titanium) was investigated. Although a significant decrease of the specular reflectivity was measured in all cases by increasing the laser fluence, the tendencies and the surface structure formations were strongly influenced by the target material and the applied gas environment. In case of the relatively large molecular mass gases (air, nitrogen and oxygen) the roughened surface became covered dominantly by nanodroplets and aggregates with filament-like structures. In case of He these structures were predominantly missing, which led to a lower darkening efficiency. The presence of oxygen had relatively strong impact on the reflective properties in case of copper and titanium. While the formation of copper-oxide enhances the darkening effect mostly at lower fluences, the formation of titanium-dioxide increases the reflectivity slowly in the high fluence range. The nitrogen atmosphere proved to be neutral for the darkening process, and at higher fluences similar darkening efficiencies were obtained for all metals which had been studied.

On the basis of the results we suppose that the use of heavy inert gasses (e.g. Xe) would probably enhance the nano-aggregate deposition without chemical side-effects and would promote the laser induced darkening of various metal surfaces.

\section{Acknowledgements}

This research was supported by the European Union and the State of Hungary, cofinanced by the European Social Fund through projects "ELITeam- Establishment of the ELI Institute at the University of Szeged: foundation of interdisciplinary research in the field of lasers and their applications" (grant no. TÁMOP-4.2.2.D-15/1/KONV-2015-0024) and the DAAD researcher exchange program (no. 56266271).

\section{References}

[1] A.Y. Vorobyev, C. Guo, Direct femtosecond laser surface nano/microstructuring and its applications, Laser Photon. Rev. 7 (2013) 385-407

[2] A.Y. Vorobyev, C. Guo, Metallic Light Absorbers Produced by Femtosecond Laser Pulses, Adv. Mech. Eng. 2010 (2010) 1-4

[3] A.Y. Vorobyev, C. Guo, Femtosecond laser nanostructuring of metals, Opt. Express. 14 (2006) 2164-2169 
[4] M.S. Ahsan, F. Ahmed, Y.G. Kim, M.S. Lee, M.B.G. Jun, Colorizing stainless steel surface by femtosecond laser induced micro/nano-structures, Appl. Surf. Sci. 257 (2011) 7771-7777

[5] A.Y. Vorobyev, C. Guo, Colorizing metals with femtosecond laser pulses, Opt. Photonics News. 19 (2008) 30-30

[6] A.Y. Vorobyev, C. Guo, Colorizing metals with femtosecond laser pulses, Appl. Phys. Lett. 92 (2008) 041914

[7] V. V. Iyengar, B.K. Nayak, K.L. More, H.M. Meyer, M.D. Biegalski, J. V. Li, et al., Properties of ultrafast laser textured silicon for photovoltaics, Sol. Energy Mater. Sol. Cells. 95 (2011) 2745-2751

[8] R. Torres, V. Vervisch, M. Halbwax, T. Sarnet, P. Delaporte, M. Sentis, Femtosecond laser texturization for improvement of photovoltaic cells: Black Silicon, J.

Optoelectron. Adv. Mater. 12 (2010) 621-625

[9] T. Sarnet, M. Halbwax, R. Torres, P. Delaporte, M. Sentis, S. Martinuzzi, et al., Femtosecond laser for black silicon and photovoltaic cells, Proc. of SPIE Vol. 6881 (2008) 688119.1-688119.15

[10] K.-E. Peiponen, T. Tsuboi, Metal surface roughness and optical reflectance, Opt. Laser Technol. 22 (1990) 127-130

[11] Y. Vorobyev, C. Guo, Shot-to-shot correlation of residual energy and optical absorptance in femtosecond laser ablation, Appl. Phys. A. 86 (2007) 235-241

[12] A.Y. Vorobyev, C. Guo, Reflection of femtosecond laser light in multipulse ablation of metals, J. Appl. Phys. 110 (2011) 043102

[13] B. Hopp, T. Smausz, T. Csizmadia, C. Vass, C. Tápai, B. Kiss, M. Ehrhardt, P. Lorenz, K. Zimmer, Production of nanostructures on bulk metal samples by laser ablation for fabrication of low-reflective surfaces, App. Phys. A, 113, (2013) 291.

[14] T. Csizmadia, T. Smausz, C. Tápai, J. Kopniczky, X. Wang, M. Ehrhardt, P. Lorenz, K. Zimmer, L. Orosz, E. Varga, A. Oszkó, B. Hopp, Comparison of the production of nanostructures on bulk metal samples by picosecond laser ablation at two wavelengths for the fabrication of low-reflective surfaces, J. Laser Micro Nanoeng. Vol. 10, No. 2, (2015) $110-118$

[15] T. Csizmadia, M. Erdélyi, T. Smausz and B. Hopp: Simulation of the reflectivity properties of microstructured titanium surface by ray tracing method, J. Laser Micro Nanoeng. Vol. 10, No. 2, (2015) 210-215

[16] P. Fan, M. Zhong, L. Li, T. Huang, H. Zhang, Rapid fabrication of surface micro/nano structures with enhanced broadband absorption on $\mathrm{Cu}$ by picosecond laser, Opt. Express. 21 (2013) 11628-11637 
[17] Y.C. Guan, W. Zhou, H.Y. Zheng, Z.L. Li, Darkening effect on AZ31B magnesium alloy surface induced by nanosecond pulse Nd:YAG laser, Appl. Surf. Sci. 280 (2013) $462-466$

[18] Y.C. Guan, W. Zhou, Z.L. Li, H.Y. Zheng, Effect of processing environment on laserinduced darkening evolution in magnesium alloy, Opt. Lasers Eng. 52 (2014) 35-40.

[19] H.X. Qian, W. Zhou, H.Y. Zheng, Study of Coloration on Excimer Laser Treated Titanium Surfaces, Surf. Rev. Lett. 15 (2008) 675-679

[20] A. Nakao, Y. Shimotsuma, M. Nishi, K. Miura, K. Hirao, Morphological control of nanoparticles by femtosecond laser irradiation, J. Ceram. Process. Res. 9 (2008) 425429.

[21] J.E. Sipe, J.F. Young, J.S. Preston, H.M. Vandriel, Laser-induced periodic surface structure. I. Theory, Phys. Rev. B Condens. Matter Mater. Phys. 27 (1983) 1141-1154.

[22] J. Long, P. Fan, M. Zhong, H. Zhang, Y. Xie, C. Lin, Superhydrophobic and colorful copper surfaces fabricated by picosecond laser induced periodic nanostructures, Appl. Surf. Sci. 311 (2014) 461-467

[23] M. Tsukamoto, K. Asuka, H. Nakano, M. Hashida, M. Katto, N. Abe, et al., Periodic microstructures produced by femtosecond laser irradiation on titanium plate, Vacuum. 80 (2006) 1346-1350

[24] N. Haustrup, G.M. O'Connor, Nanoparticle Generation During Laser Ablation and Laser-Induced Liquefaction, Phys. Procedia. 12 (2011) 46-53.

[25] Z. Lin, L. V. Zhigilei, Electron-phonon coupling and electron heat capacity of metals under conditions of strong electron-phonon nonequilibrium, Phys. Rev. B - Condens. Matter Mater. Phys. 77 (2008) 075133

[26] Peter Atkins, Physical Chemistry, 6th edition, W.H. Freeman and Company, New York, 1997

[27] A.J. Bard, L.R. Faulkner, Electrochemical Methods. Fundamentals and Applications, 2nd edition, John Wiley and Sons Inc, 2001

[28] P. Vanýsek, Electrochemical Series, in: W.M. Haynes, Handbook of Chemistry and Physics: 93rd edition, Chemical Rubber Company, 2009, pp. 5-80.

[29] A.J. Bard, R. Parsons, J. Jordan, Standard Potentials in Aqueous Solutions, Marcel Dekker, New York, 1985

[30] T. Itina, K. Gouriet, Mechanisms of Nanoparticle Formation by Laser Ablation, in: F.J. Duarte (Ed.), Laser Pulse Phenomena and Application, InTech, 2010: pp. 309-322.

[31] M. Povarnitsyn, V. Fokin, A. Voloshko, L. Delfour, T.E. Itina, Numerical Analysis of Ultrashort Laser Ablation: Application for Fabrication of Nanoparticles and Nanostructures, HPLA 2014 conference, AIP Conference Proceedings 2014, (2014) 8. 
[32] S.B. Wen, X. Mao, R. Greif, R.E. Russo, Experimental and theoretical studies of particle generation after laser ablation of copper with a background gas at atmospheric pressure, J. Appl. Phys. 101 (2007) 123105 
Figure captions

Figure 1. Normalized reflectivities of the irradiated silver (a), gold (b), copper (c), and titanium (d) samples at $550 \mathrm{~nm}$ as a function of the applied laser fluence. The samples were irradiated with 100 laser pulses in different gas ambiences.

Figure 2. High magnification (50kX) scanning electron microscopic images on silver sample irradiated with different laser fluences $(F)$ in air, $\mathrm{O}_{2}$ and $\mathrm{He}$ ambient gases. The width of the images corresponds to $\sim 2.5 \mu \mathrm{m}$ for $50 \mathrm{kX}$ and $\sim 12.5 \mu \mathrm{m}$ for the lower magnification inserts.

Figure 3. 50kX SEM images showing morphological progress of the nanostructures on copper surface irradiated with different laser fluences $(F)$ in air, $\mathrm{O}_{2}$ and $\mathrm{He}$ ambient gases. The width of the images corresponds to $\sim 2.5 \mu \mathrm{m}$ for $\times 50 \mathrm{k}$ and $\sim 12.5 \mu \mathrm{m}$ for the lower magnification inserts.

Figure 4. 50kX SEM images showing the titanium surface structures obtained with different laser fluences $(F)$ and gas environments. The width of the images corresponds to $\sim 2.5 \mu \mathrm{m}$ for $50 \mathrm{kX}$ and $\sim 12.5 \mu \mathrm{m}$ for the lower magnification inserts. 


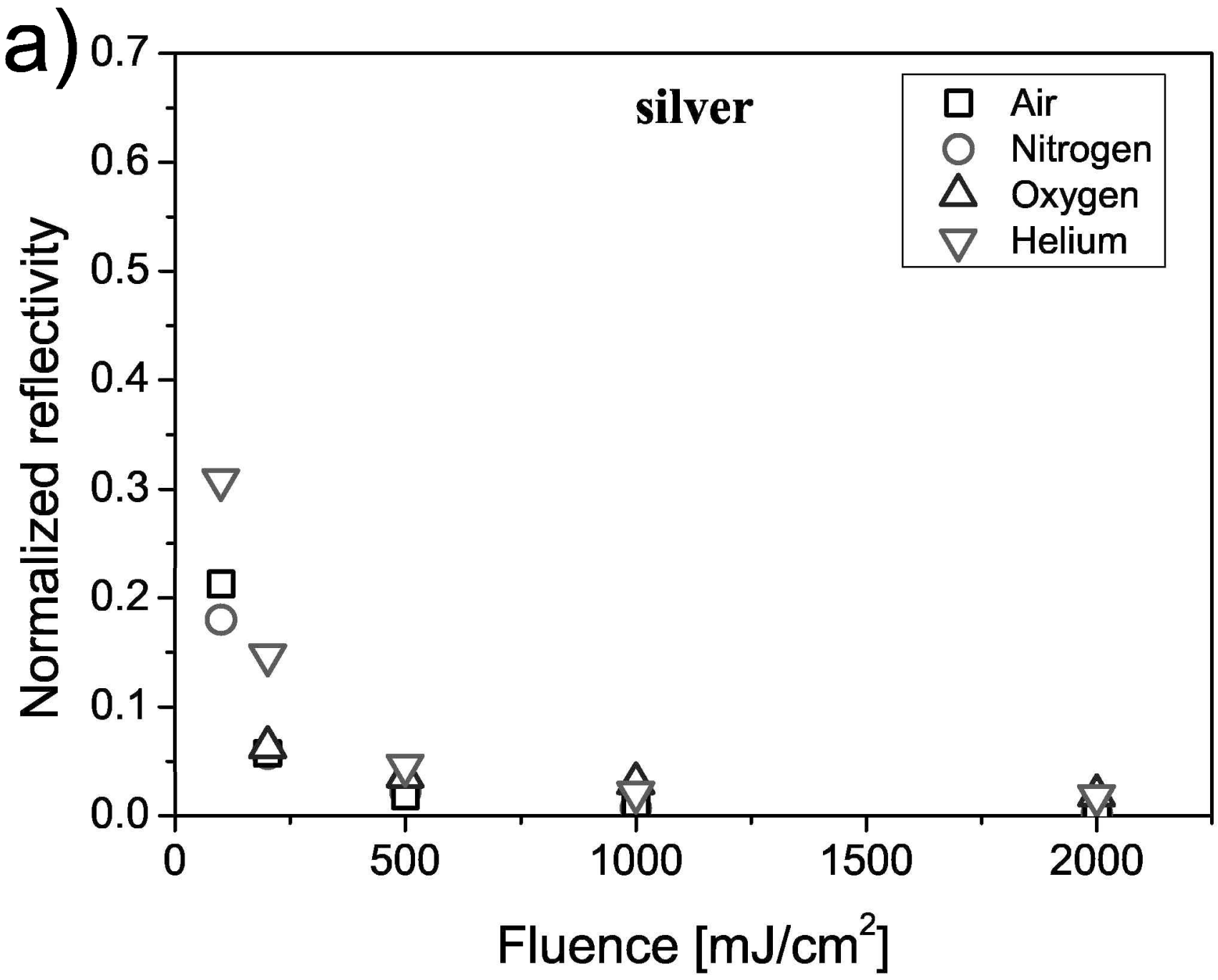




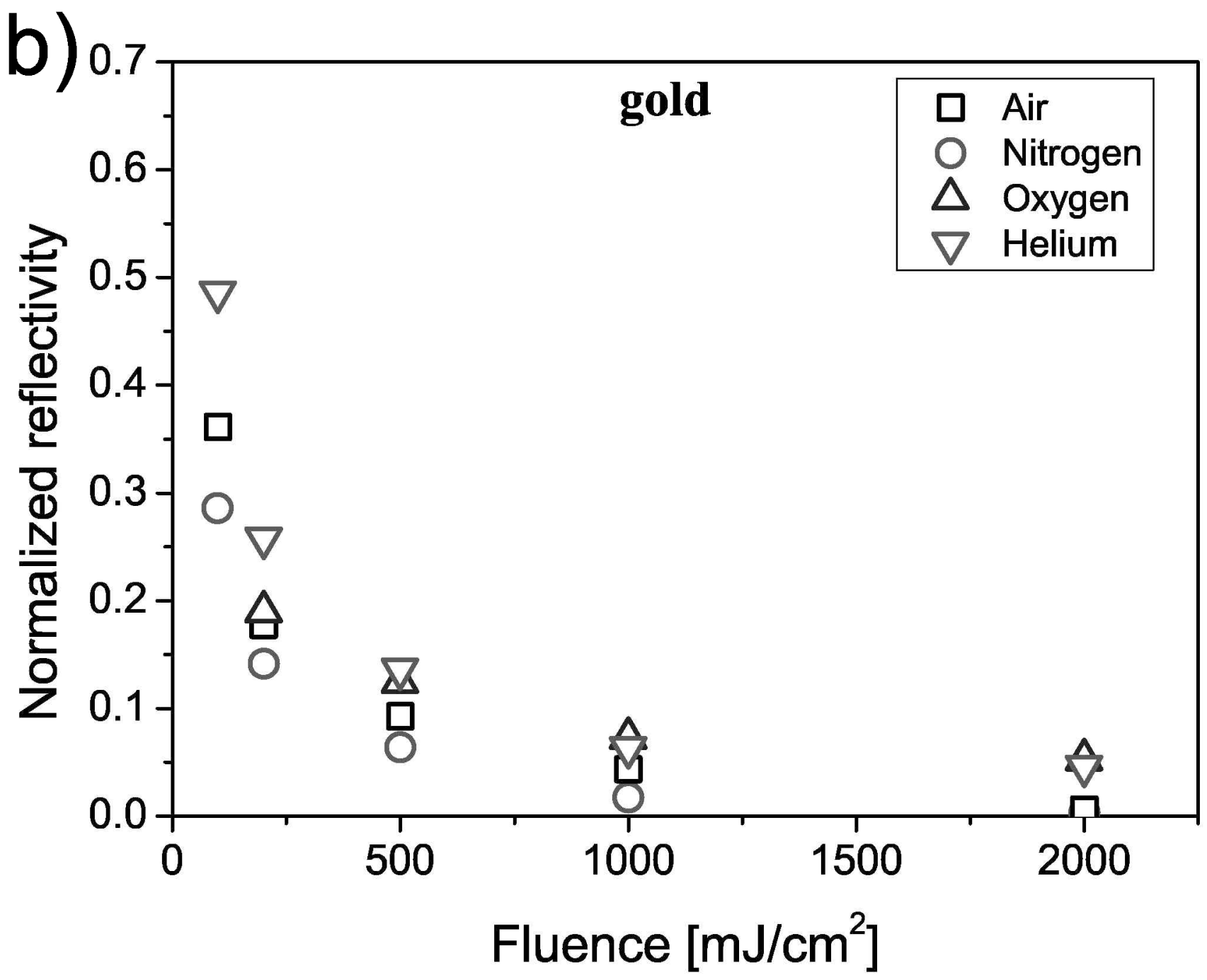




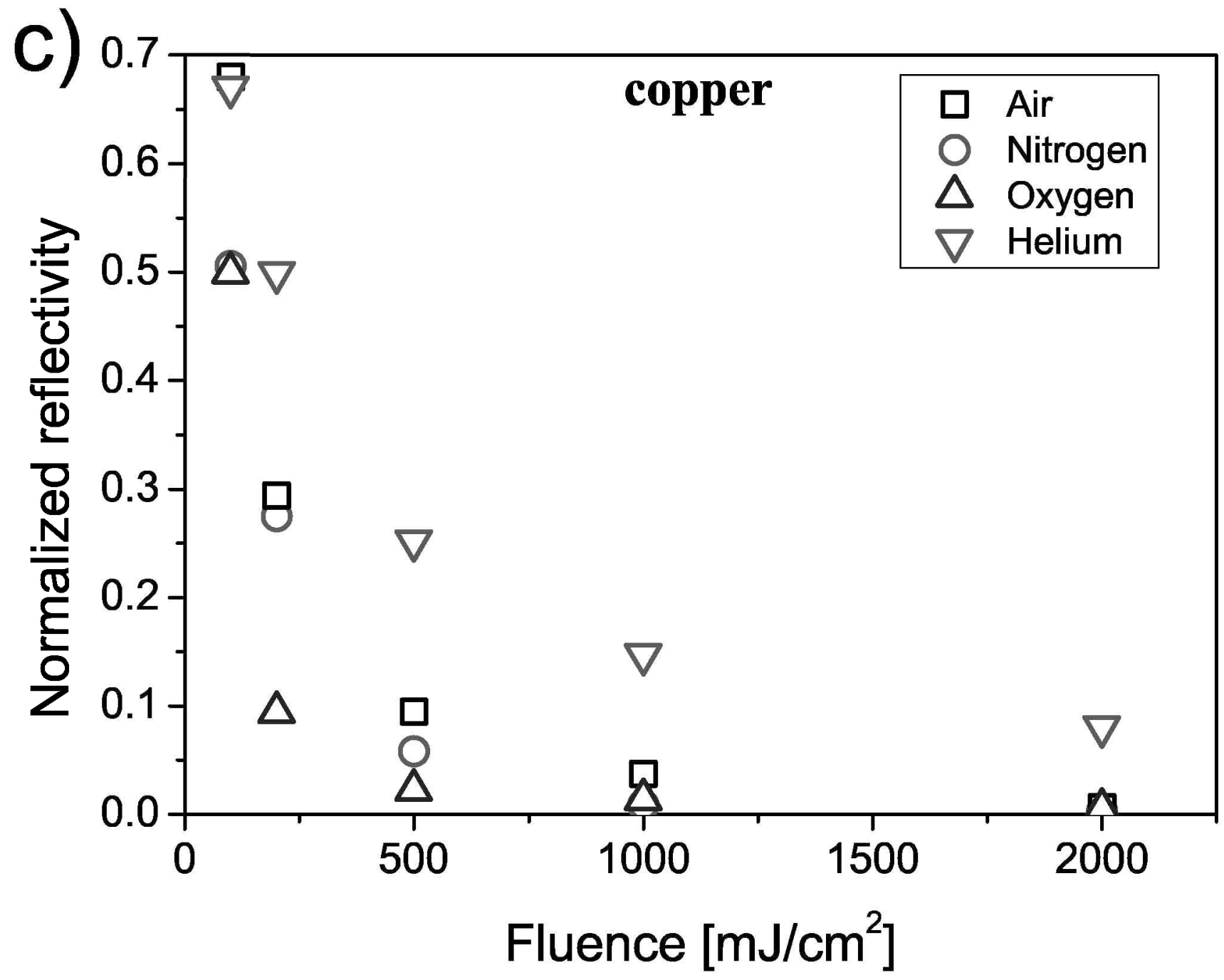




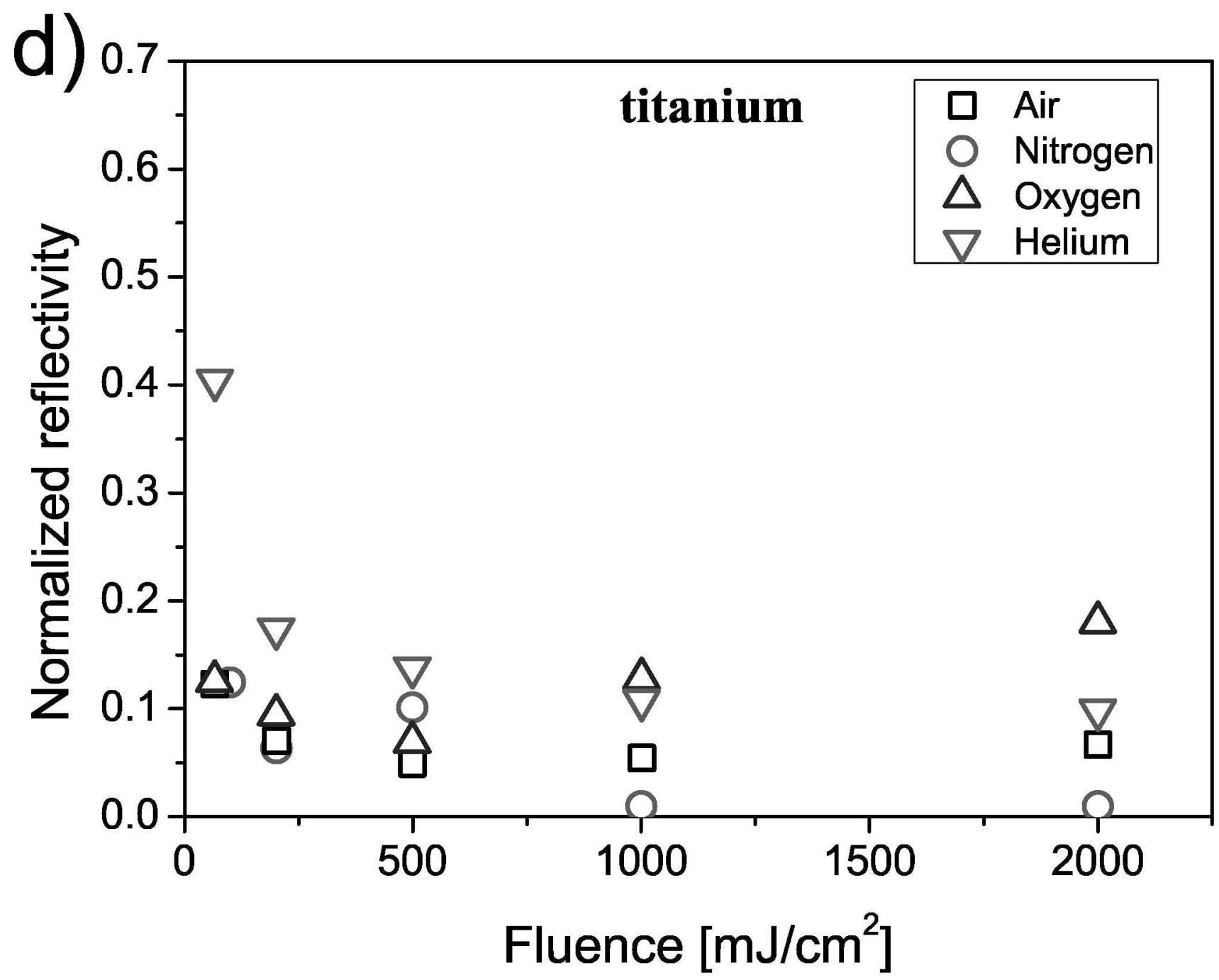


$=2000 \mathrm{~mJ} / \mathrm{cm}^{2}$
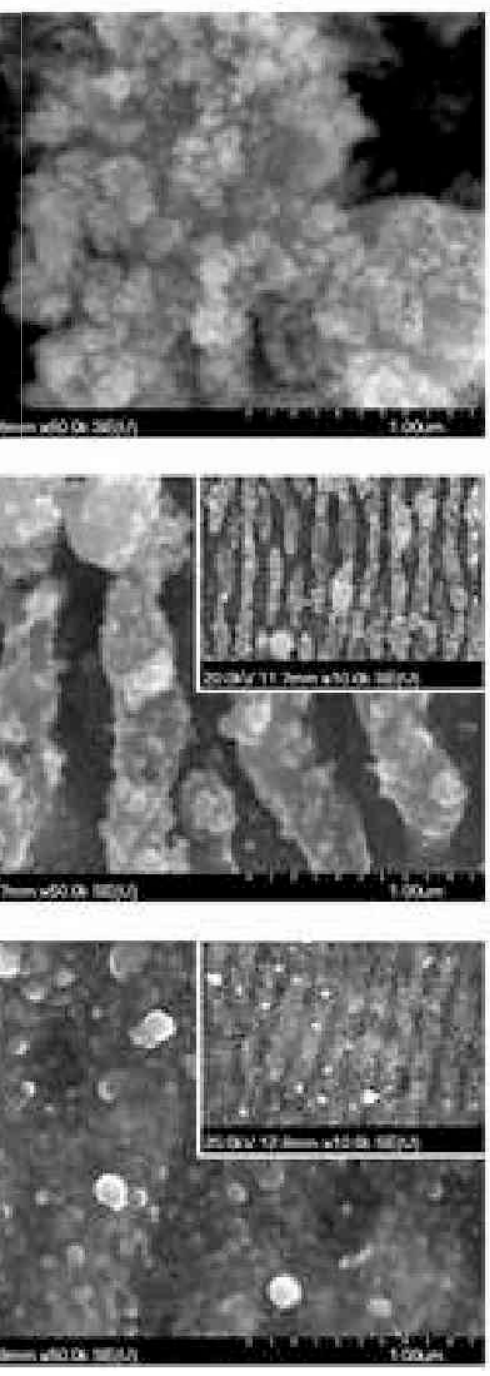

$500 \mathrm{~mJ} / \mathrm{cm}^{2}$
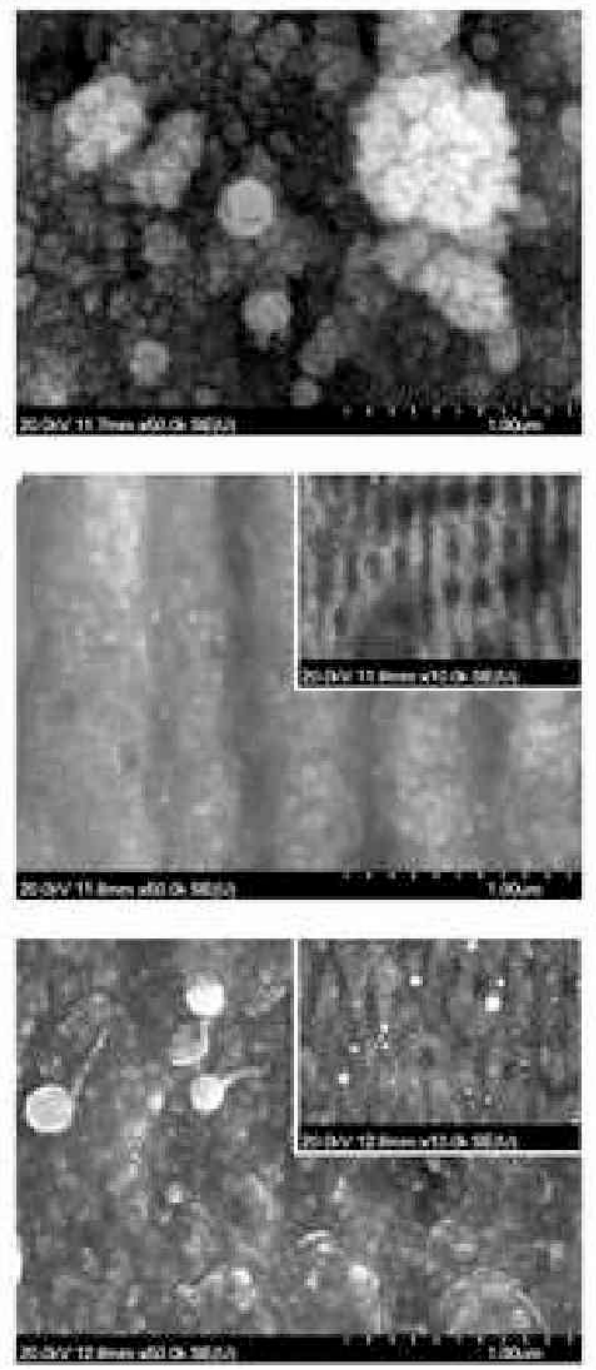

Untreat
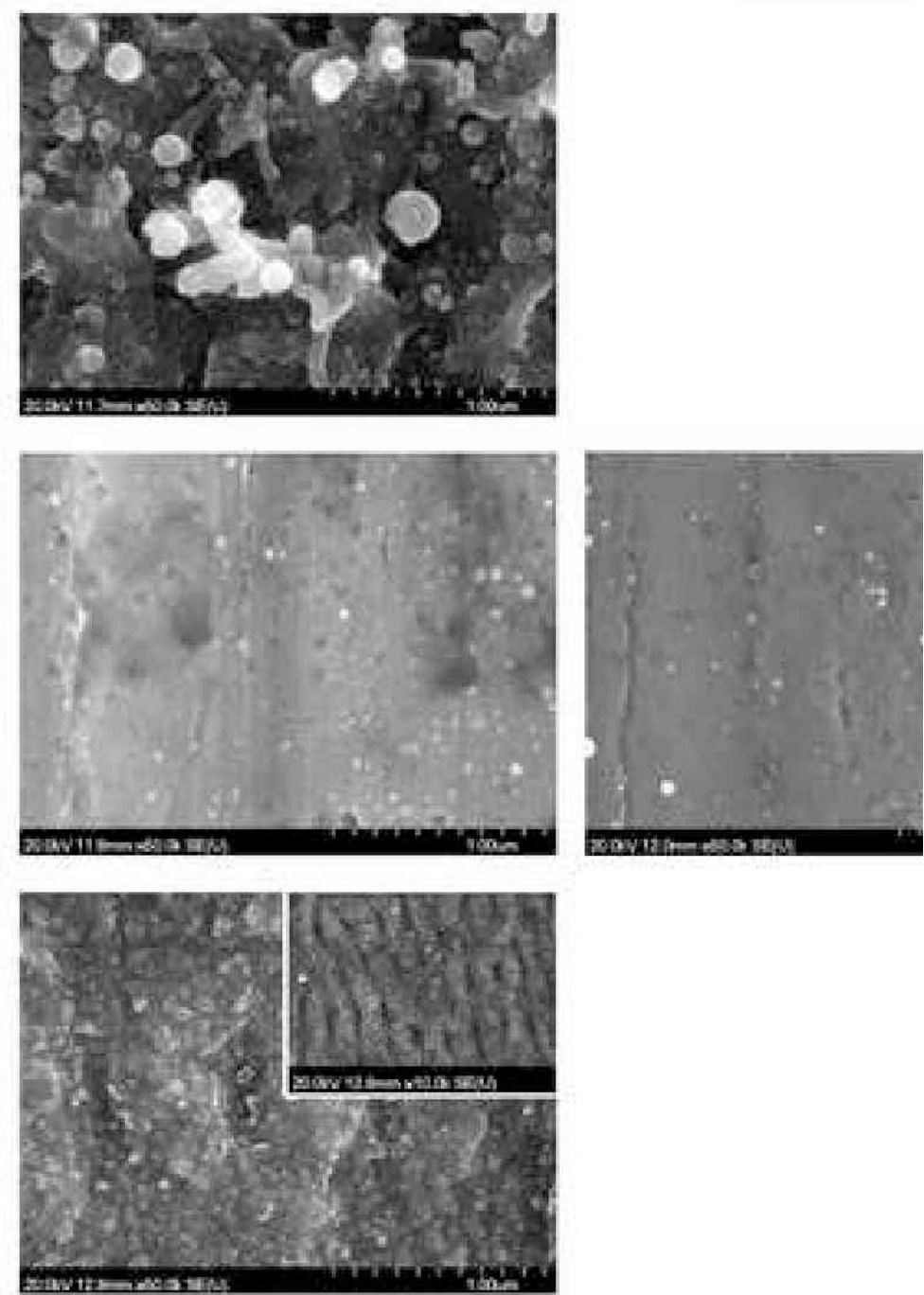
